Z. klin. Chem. u. klin. Biochem.

9. Jg., S. 471-477, November 1971

\title{
Chronische hepatische Porphyrien
}

\author{
Von M. Doss') \\ Abteilung für Klinische Biochemie am Hygiene-Institut der Pbilipps-Universität Marburg/Labn \\ D. LOOK, H. HENNING \\ Kurklinik Föbrenkamp der Bundesversicherungsanstalt für Angestellte in Mölln|Lauenburg \\ C. J. LüDERS \\ Pathologisches Institut des Städtischen Wenckebacblerankenbauses Berlin
}

\author{
W. Dölle und G. STROHMEYer \\ Medizinische Universitätsklinik Marburg/Labn
}

(Eingegangen am 8. Juni 1971)

\begin{abstract}
32 von 61 Patienten (53 Männer, 8 Frauen) mit chronischen Lebererkrankungen wie aggressive chronische Hepatitis, Fettleber, Cirrhose und Siderose, darunter 9 mit Hautsymptomen, die auf eine chronische Porphyrie hinweisen, schieden im Urin vermehrt Uro- und Koproporphyrin, Hepta-, Hexa- und Pentacarboxyporphyrine aus. Bei 21 dieser Patienten wurden die Porphyrine im Biopsiegewebe der Leber untersucht, in der Uro- und Heptacarboxyporphyrine regelmäßig und Hexacarboxyporphyrin gering nur bei höheren Konzentrationen der ersteren akkumuliert waren. Ein positiver Porphyria cutanea tarda-Index der Urinporphyrine bestätigte bei 9 Patienten das Vorliegen einer Porphyria cutanea tarda. Bei 23 Patienten bestand eine chronische hepatische Porphyrie ohne klinische Symptome, die nach dem Porphyrinanstieg in der Leber, dem Grad der Porphyrinurie und der relativen Verteilung von Uro- und Koproporphyrin, Hepta-, Hexaund Pentacarboxyporphyrinen im Urin in die Typen A, B und C differenziert wurde. Von Typ A über Typ B zu Typ C ändert sich der Quotient Kopro-/Uroporphyrin von ab 8 bis zu 0,1 simultan mit der Konstellation der Urinporphyrine und der Erhöhung der Gesamtausscheidung von 0,2 bis zu $1 \mathrm{mg} / 24$ Stunden.

Eine Porphyria cutanea tarda entwickelt sich aus einer klinisch inapparenten chronischen hepatischen Porphyrie, die selbst über Jahre stationär bleiben kann und nicht in jedem Fall in eine Porphyria cutanea tarda fortschreitet. Die chronischen hepatischen Porphyrien bei chronischen Lebererkrankungen sind die am häufigsten vorkommenden hepatischen Porphyrien.
\end{abstract}

\section{Chronic bepatic porphyrias}

An increased urinary excretion of uro- and coproporphyrin and hepta-, hexa- and pentacarboxyporphyrins was found in 32 out of 61 patients (53 men, 8 women) with chronic liver illnesses like agressive chronic hepatitis, fatty liver, cirrhosis and siderosis, of which 9 showed dermal symptoms of a chronic porphyria. In 21 of these patients, an investigation of the porphyrins in biopsy tissue of the liver generally showed an accumulation of uro- and heptacarboxyporphyrins with small amounts of hexacarboxyporphyrin, only when the concentration of the former was very high. In 9 patients the presence of a porphyria cutanea tarda was shown by a positive porphyria cutanea tarda index. 23 patients had chronic hepatic porphyrias without clinical symptoms and these were differentiated into types A, B and $C$, according to the porphyrin increase in the liver, the degree of the porphyrinuria and the relative distribution of uro-and coproporphyrin, hepta-, hexa- and pentacarboxyporphyrins in the urine. From type A to B to C, the ratio copro-/uroporphyrin changed from 8 to 0.1 with simultaneous constellation of the urinary porphyrins and an increase in the total excretion from 0.2 to $1 \mathrm{mg} / 24 \mathrm{~h}$.

A porphyria cutanea tarda develops from a clinically non-apparent chronic hepatic porphyria, which may remain stationary for years and not necessarily progress to a porphyria cutanea tarda. Chronic hepatic porphyrias in chronic liver illnesses are the most frequently occurring hepatic porphyrias.

In einer vorausgegangenen Arbeit haben wir die hepatischen Porphyrinurien als biochemisches Symptom einer Störung der Porphyrinsynthese in der Leber bei akuter intermittierender Porphyrie und Porphyria cutanea tarda differenziert und Befunde erhoben, aus denen ein flieBender Übergang von den sekundären bzw. symptomatischen Prophyrinurien bei Lebererkrankungen zu den erworbenen bzw. konstitutionellen hepatischen Porphyrien postuliert wurde (1). Damit wird gesagt, daß eine sekundäre Prophyrinurie in eine primäre hepatische Porphyrie übergehen kann. Der Übergang ist Folge einer progredienten Störung der hepatischen Porphyrinsynthese. Die bei Fettleber (1) und Lebercirrhose (2) häufig beobachtete Koproporphyrinurie ist sekundär bzw. sympto-

1) Teile dieser Arbeit wurden auf der International Conference on Porphyrin Mctabolism and the Porphyrias, Kapstadt 1970, und auf dem Congressus Biochimize Clinicae, Prag 1971, vorgetragen. matisch in bezug auf die Leberschädigung. Die Porphyrinsynthesestörung kann dann als primär angesehen werden, wenn sie, obwohl auf dem Boden einer Leberschädigung wahrscheinlich erworben, eine eigenständige Entwicklung genommen hat und nimmt, die durch permanent veränderte Aktivitäten eines oder mehrerer Enzyme der Porphyrinbiosynthesekette molekular fixiert ist und durch verschiedene Faktoren, unter denen Art und Verlauf des hepatozellulären Schadens nur einer ist, beeinflußt wird. Eine erworbene hepatische Porphyrie hat sich entwickelt, wenn die Leber Heptacarboxyporphyrin und abnorm hohe Konzentrationen von Uroporphyrin enthält (3).

Die sog. hepatische Spätporphyrie, die Porphyria cutanea tarda, wird als Endphase einer Entwicklung aufgefaßt, die über verschiedene klinisch okkulte Stadien verläuft, welche wiederum, soweit wir heute sehen, selbst 
über längere Zeit stationär bleiben können und eine eigene Gruppe hepatischer Porphyrinsynthesestörungen repräsentieren: Die chronischen hepatischen Porphyrien ohne klinische Symptome (4). Diese sind, wie die Porphyria cutanea tarda, immer mit einem Leberschaden assoziiert und wurden dadurch entdeckt, daß Leberzylinder, die eine kleinstfleckige, häufig auch nur an ein oder zwei Stellen lokalisierte Rotfluoreszenz unter langwelligem UV-Licht zeigten, zusammen mit dem Urin des Patienten auf Porphyrine untersucht wurden (5). Anhand von Bestimmungen der Porphyrine mit 8 bis 4 Carboxylgruppen im Urin, Uro- und Koproporphyrin sowie Heptacarboxy-, Hexacarboxy: und Pentacarboxyporphyrine sowie von Uroporphyrin und Heptacarboxyporphyrin in der Leber ließen sich drei Typen der klinisch symptomlosen, chronischen hepatischen Porphyrien unterscheiden, die als Typ A, B und C bezeichnet wurden (4). Ihre biochemische Differenzierung wird in der vorliegenden Arbeit dargelegt. Die Untersuchungen haben zum Ziel, chronische hepatische Porphyrinsynthesestörungen in klinisch inapparenten Stadien zu erfassen, um durch im einzelnen noch zu entwickelnde therapeutische Maßnahmen ihrer potentiellen Progression entgegenzuwirken. Es war zunächst erforderlich, diagnostische Kriterien zu erarbeiten.

\section{Patienten und Methodik}

Von November 1969 bis März 1971 wurden bei 61 chronisch leberkranken Patienten (53 Männer, 8 Frauen) die Porphyrine im Urin und in den meisten Fällen auch im Lebergewebe untersucht. Abgesehen von einer systematischen Studie über die Porphyrine in Leber und Urin bei Lebercirrhose (2) gelangte aus dem gesamten Krankengut der Klinik innerhalb des o. g. Zeitraums nur von solchen Patienten Untersuchungsmaterial zur Porphyrinanalyse, bei denen aufgrund eines unter dem langwelligen UV-Licht $(366 \mathrm{~nm})$ häufig nur geringgradig und partiell rotfluoreszierenden Leberzylinders und eines positiven oder fraglichpositiven Porphyrinfluoreszenz-Nachweises im Urin (6) von klinischer Seite der Verdacht auf eine hepatische Porphyrie erhoben wurde. Biopsiematerial aus der Leber wurde in der Regel während einer Laparoskopie entnommen und in Ausnahmen durch eine Leberblindpunktion erhalten.

\section{Vorbereitung der Urinproben}

Zur Analyse gelangte Urin, der lichtgeșchützt und kühl gesammelt und anschließend versandt wurde. Sofern die Bestimmungen nicht unmittelbar nach Eingang des Urins durchgeführt wurden, bewahrten wir den Urin, eingestellt auf $\mathrm{pH} 6-7$, bei -18 bis $-25^{\circ}$ auf. Unter diesen Bedingungen waren bei wiederholten Messungen (Standardproben) innerhalb von 3 Jahren keine Konzentrationsänderungen der Porphyrine im Urin feststellbar. Der Urin wurde direkt vor der Untersuchung auf Porphyrine mit Essigsäure auf $\mathrm{pH} 4$ eingestellt (Koṇtrolle mit Indikatorpapier; $\pm 0,2-0,3$ Einheiten). Je nach dem Ausfall des Fluoreszenz-Screeningtestes (6), der zunächst mit jedem Urin durchgeführt wurde, betrug das Probenvolumen 10 oder $20 \mathrm{ml}$ für die quantitative Bestimmung.

Darstelling der Porphyrinmetbylester und Dünnschicbtchromatographie Die freien Porphyrinsäuren im Urin wurden an Talk adsorbiert und nach Trocknung der Sorptionsschicht mit Methanol-Schwefelsäure verestert. Dabei oxydierten auch Porphyrinogene zu Porphyrinen. Die Porphyrinmethylester wurden mit Chloroform extrahiert (7). Versuche, bei denen sowohl der saure als auch der neutralisierte Chloroformextrakt mit Porphyrinmethylestern (aus Urin, Leber, Mikroorganismen) gegen eine wäßr. Jodlösung
$(0,5 \mathrm{mg} / \mathrm{l})$ aúsgeschüttelt wurde, ergaben weder qualitative Unterschiede in der Zusammensetzung der Porphyringemische noch quantitative Differenzen.

Aus Leberzylindern wurden die Porphyrine ebenfalls als Methylester dargestellt (8).

Dic Trennung der Porphyrinmethylester erfolgte auf fertigen Kieselgel-Aluminium-Dünnschichtkarten (7). Hierzu sind weitere Einzelheiten in den Legenden zu den Abbildungen 1 bis 3 aufgeführt.

\section{Spektrophotometriscbe und fuorometriscbe Analyse der Porpbyrine}

Waren auf den Leberporphyrin-Chromatogrammen, infolge von wenigen Milligramm Lebergewebe als Ausgangsmaterial, nur sehr kleine Mengen an Porphyrinen vorhanden, wurden sie direkt im Chromatogramm fluorometrisch gemessen (9). Im übrigen wurden die Porphyrinmethylester eluiert und spektrophotometrisch entweder in Chloroform (7) oder, bei Vorliegen in kleinen Konzentrationen, nach Umwandlung in ihre Zinkchelate als solche bestimmt (Abb. 1) (10). Meßgröße wat die Extinktion am Soret-Maximum $(7,10)$, aus der mit Hilfe millimolarer Extinktionskoeffizienten die Konzentration bereçhnet wurde (Tab. 1). Hexa- und Pentacarboxyporphyrin-Methylester aus dem Urin wurden häufig, die Porphyrinmethylester aus den Leberzylindern immer als Zinkchelate analysiert.

Tab. 1

Millimolare Extinktionskoeffizienten der Porphyrin-Methylester bzw. deren Zinkchelate am Soret-Maximum

\begin{tabular}{lcc}
\hline Porphyrinmethylester aus & $\begin{array}{c}\varepsilon\left(1 \cdot \mathrm{mMol}^{-1} \cdot \mathrm{cm}^{-1}\right) \\
\text { als Zinkchelat }\end{array}$ \\
\hline Uroporphyrin & 216 & 406 \\
Heptacarboxyporphyrin & 207 & 393 \\
Hexacarboxyporphyrin & 198 & 380 \\
Pentacarboxyporphyrin & 189 & 366 \\
Koproporphyrin & 180 & 353 \\
\hline
\end{tabular}

\section{Bestimmung von $\delta$-Aminolävulinsäure und Porphobilinogen}

$\delta$-Aminolävulinsäure und Porphobilinogen.im Urin wurden nach ionenaustauschchromatographischer Trennung auf Fertigsäulen spektrophotometrisch bestimmt (11).

\section{Beurteilung der Urinfarbe}

Die Urinfarbe läßt, wie .auch schon andere Untersucher $(12,13)$ beobachtet haben, logische Schlüsse auf den Gehalt an Porphobilinogen und/oder Porphyrinen nicht zu. Porphobilinogen ist eine farblose Substanz. Die Urinfarbe hängt jedoch nicht nur von Farbverbindungen, sondern auch von physikalischen Faktoren ab (12). Nach unseren Erfahrungen können normalfarbene Urine verhältnismäßig hohe Porphyrinkonzentrationen enthalten (bis $3 \mathrm{mg} / \mathrm{l}$ ); umgekehrt können in dunklen, rot- oder mahagonifarbenen Urinen Porphyrine nur gering exhöht sein. Ehe ein Urin mit in Lösung befindlichen Porphytinen die typische Rotfluoreszenz im langwelligen UV-Licht zeigt, müssen sehr hohe Porphyrinkonzentrationen vorhanden sein $(>4 \mathrm{mg} / \mathrm{l})$. Außerdem ist die Fluoreszenz vom pH des Urins abhängig. Besser jedoch als die UV-Exposition des Urins eignet sich ein Fluoreszenzsuchtest (6), der eine erhöhte Porphyrinkonzentration im Urin anzeigt.

\section{Ergebnisse}

Von 61 Patienten mit chronischem Leberschaden hatten 32 eine erworbene hepatische Porphyrie. Hierbei handelt es sich in 23 Fällen um eine chronische hepatische Porphyrie ohne klinische Symptome und in 9 um eine Porphyria cutanea tarda. Die Ergebnisse sind in den Tabellen 2 bis 6 enthalten. Charakteristische Befunde werden in den Abbildungen 1 bis 5 wiedergegeben.

Bei allen Patienten war die Lebererkrankung klinisch, laparoskopisch und histologisch diagnostiziert worden. Die in den Tabellen 2 bis 5 aufgeführten Diagnosen 
beruhen auf den histologisch gesicherten Befunden. Die Leber war palpatorisch in allen Fällen vergrößert. Alkoholkonsum wurde von nur wenigen Patienten verneint; bei einigen lag er über $100 \mathrm{~g} / \mathrm{Tag}$. Das Albumin/Globulin-Verhältnis im Serum war regelmäßig erniedrigt. Eine
Korrelation zwischen den laboratoriumsmedizinischen Daten einschließlich des Bromsulfophthaleintests und den Porphyrinen in Leber und Urin wurde nicht gefunden. Außer den Patienten mit Porphyria cutanea tarda (Tab. 5) wiesen die Leberkranken (Tab. 2-4) keine für eine

Tab. 2

Urinporphyrine bei chronischer hepatischer Porphyrie Typ A

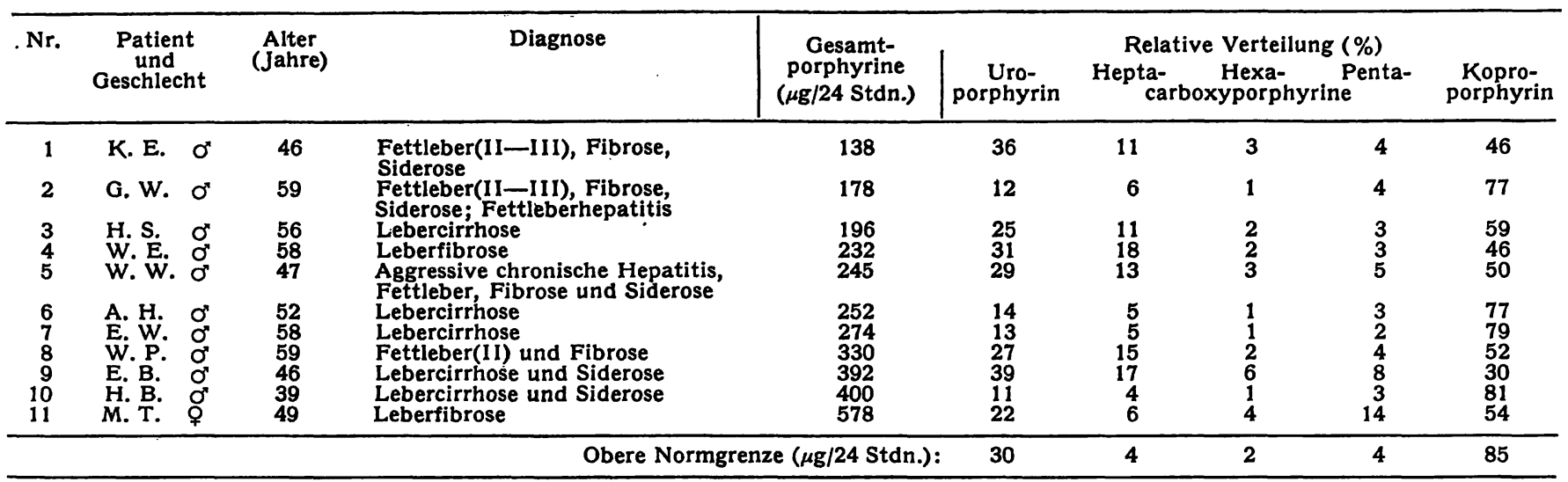

Tab. 3

Urinporphyrine bei chronischer hepatischer Porphyrie Typ B

\begin{tabular}{|c|c|c|c|c|c|c|c|c|c|}
\hline $\mathrm{Nr}$. & $\begin{array}{l}\text { Patient } \\
\text { und } \\
\text { Geschlecht }\end{array}$ & $\begin{array}{c}\text { Alter } \\
\text { (Jahre) }\end{array}$ & Diagnose & $\begin{array}{c}\text { Gesamt- } \\
\text { porphyrine } \\
(\mu \mathrm{g} / 24 \text { Stdn. })\end{array}$ & $\underset{\text { porphyrin }}{\text { Uro- }}$ & $\begin{array}{c}\text { Relat } \\
\text { Hepta- } \\
\text { carb }\end{array}$ & $\begin{array}{l}\text { Verte } \\
\text { Hexa } \\
\text { xyporp }\end{array}$ & $\begin{array}{l}(\%) \\
\text { Penta- } \\
\text { e }\end{array}$ & $\begin{array}{c}\text { Kopro- } \\
\text { porphyrin }\end{array}$ \\
\hline $\begin{array}{l}12 \\
13\end{array}$ & $\begin{array}{l}\text { F. R. } \quad \sigma^{\prime \prime} \\
\text { K. R. } \quad \sigma^{\prime \prime}\end{array}$ & $\begin{array}{l}53 \\
57\end{array}$ & \multirow{6}{*}{$\begin{array}{l}\text { Lebercirrhose, Siderose } \\
\text { Aggressive chronische Hepatitis } \\
\text { mit cirrhotischem Umbau, Siderose } \\
\text { Aggressive chronische Hepatitis, } \\
\text { Fibrose und Siderose } \\
\text { Lebercirrhose, Siderose } \\
\text { Cirrhose mit starker entzündlicher } \\
\text { Aktivität, Siderose } \\
\text { Fettleberhepatitis, Fibrose, } \\
\text { Siderose } \\
\text { Aggressive chronische Hepatitis, } \\
\text { Fibrose und Siderose } \\
\text { Aggressive chronische Hepatitis } \\
\text { mit cirrhotischem Umbau }\end{array}$} & $\begin{array}{l}201 \\
214\end{array}$ & $\begin{array}{l}38 \\
56\end{array}$ & $\begin{array}{l}23 \\
18\end{array}$ & $\begin{array}{l}6 \\
3\end{array}$ & $\begin{array}{r}10 \\
4\end{array}$ & $\begin{array}{l}23 \\
19\end{array}$ \\
\hline 14 & P. F. O & 53 & & 236 & 62 & 14 & 2 & 3 & 19 \\
\hline $\begin{array}{l}15 \\
16\end{array}$ & $\begin{array}{ll}\text { W. F. } & \sigma^{\prime \prime} \\
\text { H. G. } & \sigma^{\prime}\end{array}$ & $\begin{array}{l}53 \\
57\end{array}$ & & $\begin{array}{l}310 \\
331\end{array}$ & $\begin{array}{l}45 \\
42\end{array}$ & $\begin{array}{l}20 \\
19\end{array}$ & $\begin{array}{l}5 \\
4\end{array}$ & $\begin{array}{l}5 \\
6\end{array}$ & $\begin{array}{l}25 \\
29\end{array}$ \\
\hline 17 & K. R. $\sigma^{*}$ & 39 & & 350 & 48 & 16 & 2 & 7 & 27 \\
\hline 18 & S. K. $\quad \sigma^{\prime \prime}$ & 59 & & 508 & 49 & 15 & 4 & 5 & .27 \\
\hline 19 & M. R. o & 60 & & 642 & 57 & 13 & 3 & 4 & 23 \\
\hline
\end{tabular}

Tab. 4

Urinporphyrine bei chronischer hepatischer Porphyrie Typ C

\begin{tabular}{|c|c|c|c|c|c|c|c|c|c|c|c|}
\hline Nr. & \multicolumn{2}{|c|}{$\begin{array}{l}\text { Patient } \\
\text { und } \\
\text { Geschlecht }\end{array}$} & $\begin{array}{c}\text { Alter } \\
\text { (Jähré) }\end{array}$ & \multicolumn{2}{|l|}{ Diagnose } & $\begin{array}{c}\text { Gesamt- } \\
\text { porphyrine } \\
(\mu \mathrm{g} / 24 \text { Stdn. })\end{array}$ & $\begin{array}{c}\text { Uro- } \\
\text { porphyrin }\end{array}$ & \multicolumn{3}{|c|}{$\begin{array}{l}\text { Relative Verteilung ( } \%) \\
\text { Hepta- Hexa- Penta- } \\
\text { carboxyporphyrine }\end{array}$} & \multirow{2}{*}{$\begin{array}{c}\begin{array}{c}\text { Kopro- } \\
\text { porphyrin }\end{array} \\
11 \\
10 \\
6 \\
12\end{array}$} \\
\hline $\begin{array}{l}20 \\
21 \\
22 \\
23\end{array}$ & $\begin{array}{l}\text { W. S. } \\
\text { R. C. } \\
\text { P. L. } \\
\text { H. T. }\end{array}$ & $\begin{array}{l}\sigma^{\prime \prime} \\
\sigma^{\prime \prime} \\
\sigma^{\prime \prime} \\
\sigma^{\prime \prime}\end{array}$ & $\begin{array}{l}61 \\
53 \\
43 \\
43\end{array}$ & $\begin{array}{l}\text { Lebercirrhose } \\
\text { Leberfibrose } \\
\text { Fettleber(III), Fibrose } \\
\text { Fettleber(III), Fibrose }\end{array}$ & $\cdot$ & $\begin{array}{l}415 \\
558 \\
621 \\
898\end{array}$ & $\begin{array}{l}58 \\
46 \\
59 \\
66\end{array}$ & $\begin{array}{l}22 \\
37 \\
26 \\
19\end{array}$ & $\begin{array}{l}4 \\
3 \\
4 \\
1\end{array}$ & $\begin{array}{l}5 \\
4 \\
5 \\
2\end{array}$ & \\
\hline
\end{tabular}

Tab. 5

Urinporphyrine bei Porphyria cutanea tarda (chronische hepatische Porphyrie Typ D)

\begin{tabular}{|c|c|c|c|c|c|c|c|c|c|}
\hline \multirow[t]{2}{*}{ Nr. } & \multirow{2}{*}{$\begin{array}{l}\text { Patient } \\
\text { und } \\
\text { Geschlecht }\end{array}$} & \multirow{2}{*}{$\begin{array}{c}\text { Alter } \\
\text { (Jahre) }\end{array}$} & \multirow[t]{2}{*}{ Diagnose } & \multirow{2}{*}{$\begin{array}{c}\text { Gesamt- } \\
\text { porphyrine } \\
(\mu \mathrm{g} / 24 \mathrm{Stdn}) .\end{array}$} & \multirow[b]{2}{*}{$\begin{array}{c}\text { Uro- } \\
\text { porphyrin }\end{array}$} & \multicolumn{3}{|c|}{ Relative Verteilung (\%) } & \multirow[b]{2}{*}{$\begin{array}{l}\text { Kopro- } \\
\text { porphyrin }\end{array}$} \\
\hline & & & & & & $\begin{array}{c}\text { Hepta- } \\
\text { car }\end{array}$ & $\begin{array}{l}\text { Hexa } \\
\text { cyporp }\end{array}$ & Penta- & \\
\hline 24 & H.S. ơ & 55 & \multirow{6}{*}{$\begin{array}{l}\text { Cirrhose mit starker entzündlicher } \\
\text { Aktivität, Siderose } \\
\text { Aggressive chronische Hepatitis, } \\
\text { Fibrose, Siderose } \\
\text { Leberfibrose, Siderose } \\
\text { Persistierende chronische Hepa- } \\
\text { titis, Siderose } \\
\text { Leberfibrose } \\
\text { Aggressive chronische Hepatitis, } \\
\text { Siderose } \\
\text { Aggressive chronische Hepatitis, } \\
\text { Fibrose } \\
\text { Aggressive chronische Hepatitis } \\
\text { Chronische Hepatitis }\end{array}$} & 780 & 53 & 32 & 4 & 5 & 6 \\
\hline 25 & C. L. O & 53 & & 1786 & 60 & 21 & 5 & 5 & 9 \\
\hline $\begin{array}{l}26 \\
27\end{array}$ & $\begin{array}{ll}\text { M. D. } & \stackrel{\text { o }}{\text { F. G. }}\end{array}$ & $\begin{array}{l}53 \\
59\end{array}$ & & $\begin{array}{l}1991 \\
2341\end{array}$ & $\begin{array}{l}64 \\
60\end{array}$ & $\begin{array}{l}22 \\
26\end{array}$ & $\begin{array}{l}4 \\
5\end{array}$ & $\begin{array}{l}4 \\
5\end{array}$ & $\begin{array}{l}6 \\
4\end{array}$ \\
\hline $\begin{array}{l}28 \\
29\end{array}$ & $\begin{array}{l}\text { W. H. O' } \\
\text { J. D. }\end{array}$ & $\begin{array}{l}48 \\
50\end{array}$ & & $\begin{array}{l}2367 \\
2459\end{array}$ & $\begin{array}{l}5 \dot{6} \\
55\end{array}$ & $\begin{array}{l}31 \\
32\end{array}$ & $\begin{array}{l}7 \\
4\end{array}$ & $\begin{array}{l}2 \\
4\end{array}$ & $\begin{array}{l}4 \\
5\end{array}$ \\
\hline 30 & o. W. $\sigma^{n}$. & 57 & & 3720 & 55 & 30 & 7 & 4 & 4 \\
\hline $\begin{array}{l}31 \\
32\end{array}$ & $\begin{array}{l}\text { T. H. }{ }^{\text {H. }} \text {. } \\
\text { E. }\end{array}$ & $\begin{array}{l}39 \\
71\end{array}$ & & $\begin{array}{l}5088 \\
8980\end{array}$ & $\begin{array}{l}55 \\
46\end{array}$ & $\begin{array}{l}26 \\
30\end{array}$ & $\begin{array}{l}7 \\
9\end{array}$ & $\begin{array}{l}5 \\
9\end{array}$ & $\begin{array}{l}7 \\
6\end{array}$ \\
\hline
\end{tabular}


Tab. 6

Porphyrine im Biopsiegewebe der Leber bel chronischer hepatischer Porphyrie Typ A, B, C einschließlich der Porphyria cutanea tarda (D)

\begin{tabular}{|c|c|c|c|c|c|c|c|c|c|c|}
\hline $\begin{array}{c}\text { Nr. der } \\
\text { Patienten }\end{array}$ & $\begin{array}{c}\text { Rotfluoreszenz } \\
\text { der } \\
\text { Leberbiopsie }\end{array}$ & $\begin{array}{l}\text { Gesamt- } \\
\text { porphyrine } \\
(\mu \mathrm{g} / \mathrm{g})\end{array}$ & $\begin{array}{l}\text { Uro- } \\
\text { porphyrin }\end{array}$ & Hepta- & $\begin{array}{l}\text { Relative Ve } \\
\text { Hexa- } \\
\text { carboxyporphyrine }\end{array}$ & $\begin{array}{l}\text { teilung } \\
\text { Penta- }\end{array}$ & $\begin{array}{c}\text { Kopro- } \\
\text { porphyrin }\end{array}$ & $\begin{array}{c}\text { Proto- } \\
\text { porphyrin }\end{array}$ & Typ & \\
\hline $\begin{array}{r}1 \\
2 \\
4 \\
5 \\
7 \\
8 \\
9 \\
11 \\
13 \\
14 \\
18 \\
19 \\
20 \\
22 \\
23 \\
24 \\
25 \\
26 \\
27 \\
31 \\
32\end{array}$ & $\begin{array}{c}\overline{ } \\
+++ \\
++ \\
(+) \\
+ \\
+ \\
+ \\
+ \\
++ \\
++ \\
+++ \\
+++ \\
+++ \\
++ \\
+++ \\
+++ \\
+++ \\
+++ \\
+++\end{array}$ & $\begin{array}{r}3 \\
3 \\
53 \\
15 \\
4 \\
5 \\
9 \\
4 \\
9 \\
4 \\
47 \\
34 \\
91 \\
67 \\
85 \\
35 \\
56 \\
413 \\
55 \\
75 \\
874\end{array}$ & $\begin{array}{l}88 \\
87 \\
71 \\
88 \\
.86 \\
83 \\
83 \\
89 \\
86 \\
87 \\
78 \\
81 \\
82 \\
. \quad 87 \\
80 \\
69 \\
70 \\
63 \\
64 \\
66 \\
62\end{array}$ & $\begin{array}{l}12 \\
13 \\
29 \\
12 \\
14 \\
17 \\
17 \\
11 \\
14 \\
13 \\
22 \\
19 \\
18 \\
13 \\
20 \\
30 \\
27 \\
36 \\
35 \\
33 \\
37\end{array}$ & $\begin{array}{l}\overline{ } \\
\pm \\
\pm \\
= \\
= \\
= \\
\bar{z} \\
+ \\
+ \\
+ \\
+ \\
1 \\
3 \\
1 \\
1 \\
1 \\
1\end{array}$ & $\begin{array}{l}= \\
= \\
= \\
= \\
= \\
= \\
= \\
= \\
= \\
=\end{array}$ & $\begin{array}{l}= \\
\pm \\
\pm \\
= \\
= \\
\pm \\
+ \\
+ \\
+ \\
+ \\
+ \\
+ \\
+ \\
+\end{array}$ & $\begin{array}{l}+ \\
+ \\
+ \\
+ \\
+ \\
+ \\
+ \\
+ \\
+ \\
+ \\
+ \\
+ \\
+ \\
+ \\
+ \\
+ \\
+\end{array}$ & $\begin{array}{l}\text { A } \\
\text { A } \\
\text { A } \\
\text { A } \\
\text { A } \\
\text { A } \\
\text { A } \\
\text { A } \\
\text { B } \\
\text { B } \\
\text { B } \\
\text { B } \\
\text { C } \\
\text { C } \\
\text { C } \\
\text { D } \\
\text { D } \\
\text { D } \\
\text { D } \\
\text { D } \\
\text { D }\end{array}$ & - \\
\hline
\end{tabular}

+ chromatographisch nachgewiesen

- im Chromatogramm nicht vorhanden

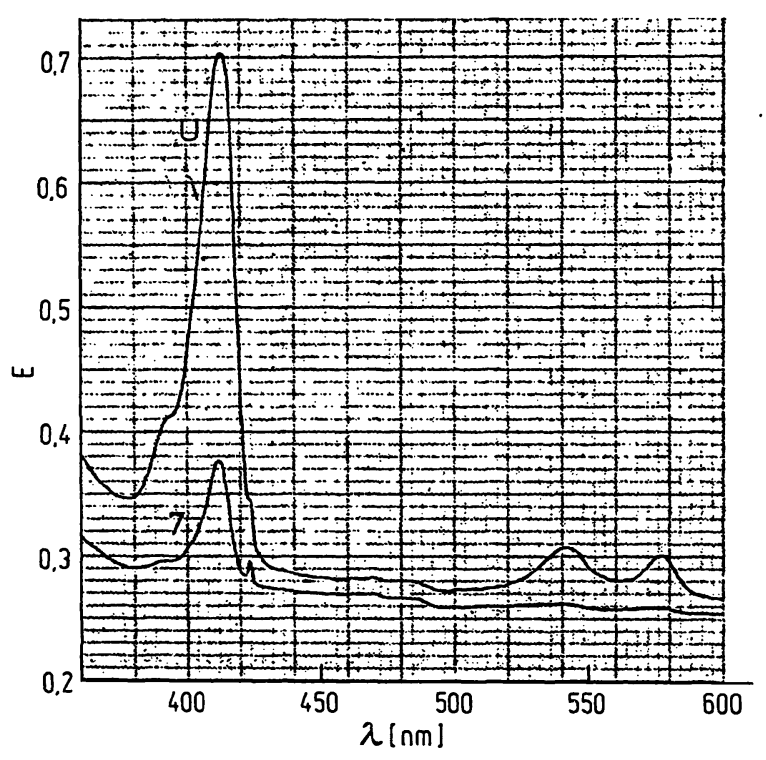

Abb. 1

Spektren der Zinkchelate von Uroporphyrin-(U) und Heptacarboxyporphyrinmethylester (7), die aus dem Leberbiopsiegewebe des 4 und 6, $\mathrm{Nr}$. 23) dünnschichtchromatographisch isoliert wurden. lumen $1 \mathrm{ml}$, Schichtdicke $2 \mathrm{~cm}$

chronische Porphyrie typischen Hauterscheinungen (14) auf. Dennoch waren bei den in den Tabellen 2 bis 4 aufgeführten Patienten der Porphyringehalt in. der Leber und die Porphyrinausscheidung im Urin erhöht (Abb. 2-4). Physiologischerweise enthält $1 \mathrm{~g}$ Leber des Erwachsenen etwa $1 \mu \mathrm{g}$ Porphyrine, unter denen Protoporphyrin bei weitem überwiegt (8); die Zusammensetzung der Leberporphyrine folgt der Regel Protoporphyrin > Koproporphyrin $>$ Uroporphyrin. Das lipophile Protoporohyrin wird mit der Galle ausgeschieden; es kommt auch unter pathologischen Bedingungen selten und dann nur in geringen Konzentrationen im Urin vor. Koproporphyrin wird überwiegend im Urin ausgeschieden, während das hydrophile Uroporphyrin unter physiologischen Bedingungen praktisch nur über die Niere ausgeschieden wird. Im Normalurin überwiegt Koproporphyrin immer gegenüber Uroporphyrin im Verhältnis von 3 bis $10 \mathrm{zu} 1$.

Wie aus den Tabellen 2 bis 4 hervorgeht, waren bei den Patienten mit chronischer Hepatitis, Cirrhose, Fettleber, Fibrose und Siderose im Urin sämtliche Porphyrine mit 8 bis 4 Carboxylgruppen erhöht und in der Leber Uro-

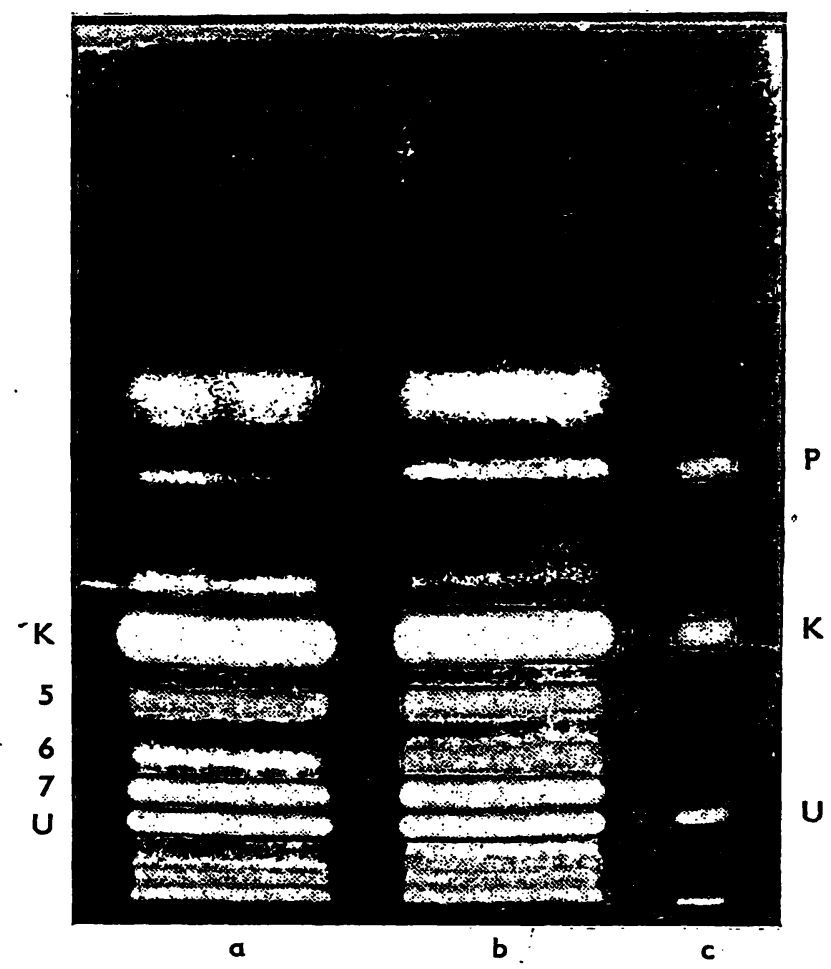

Abb. 2

Fluoreszenzaufnahme eines Dünnschichtchromatogramims mit Porphyrinen aus je $20 \mathrm{ml}$ Urin der Patienten W. P. (a) und E. B. (b) mit chronischer hepatischer Porphyrie Typ A. (Tab. 2; Nr. 8 und 9) Auf Bahn c Vergleichssubstanzen von Uro-, Kopro- und Protoporphyrinmethylester: $U$, $K$ und $P .7,6$ und $5=$ Hepta-, Hexa- und Pentacarboxyporphyrinmethylester. Chromatographie an Kieselgel A Aluminium-Fertigfolien 150 (Schlecher \& Schüll) im Lösungsmittel system Benzol-Essigsaureathylester-Methanol $(85+13,5+1,5$ von Proper um Porphorine hellblau fluoreszieren) 


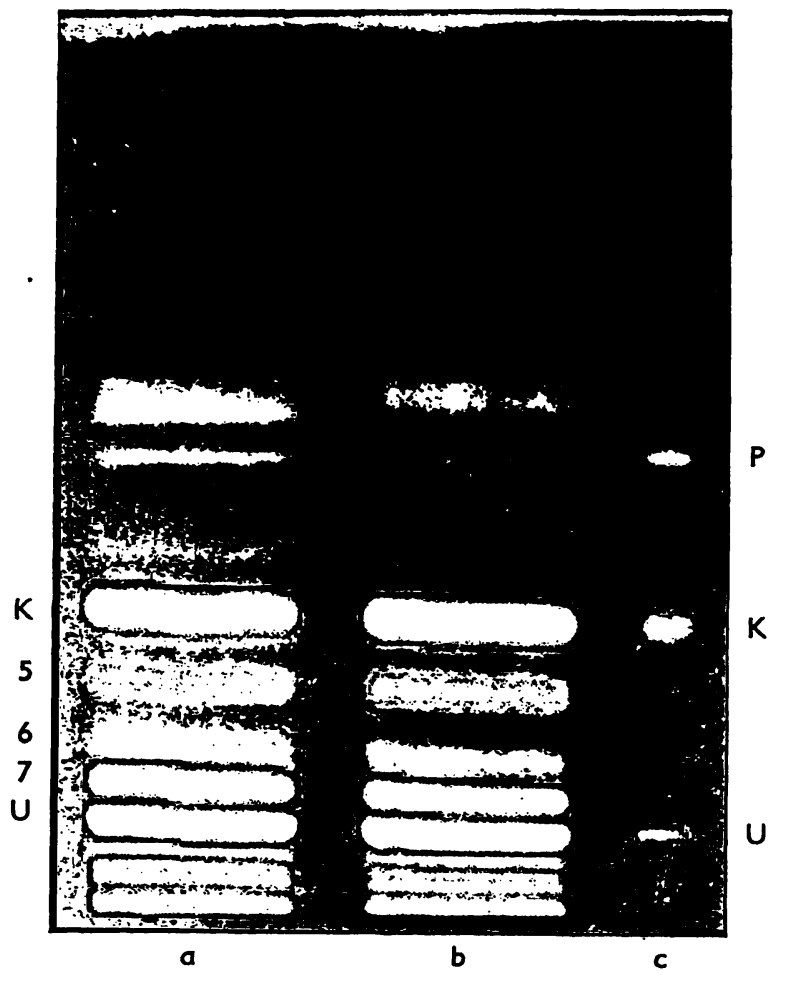

Abb: 3

Dünnschichtchromatogramm mit Urinporphyrinen der Patienten M. R. (a) und S. K. (b) mit chronischer hepatischer Porphyrie Typ B c. Für experimentelle Details und Erklärung der Abkürzungen siehe Legende zur Abbildung 2

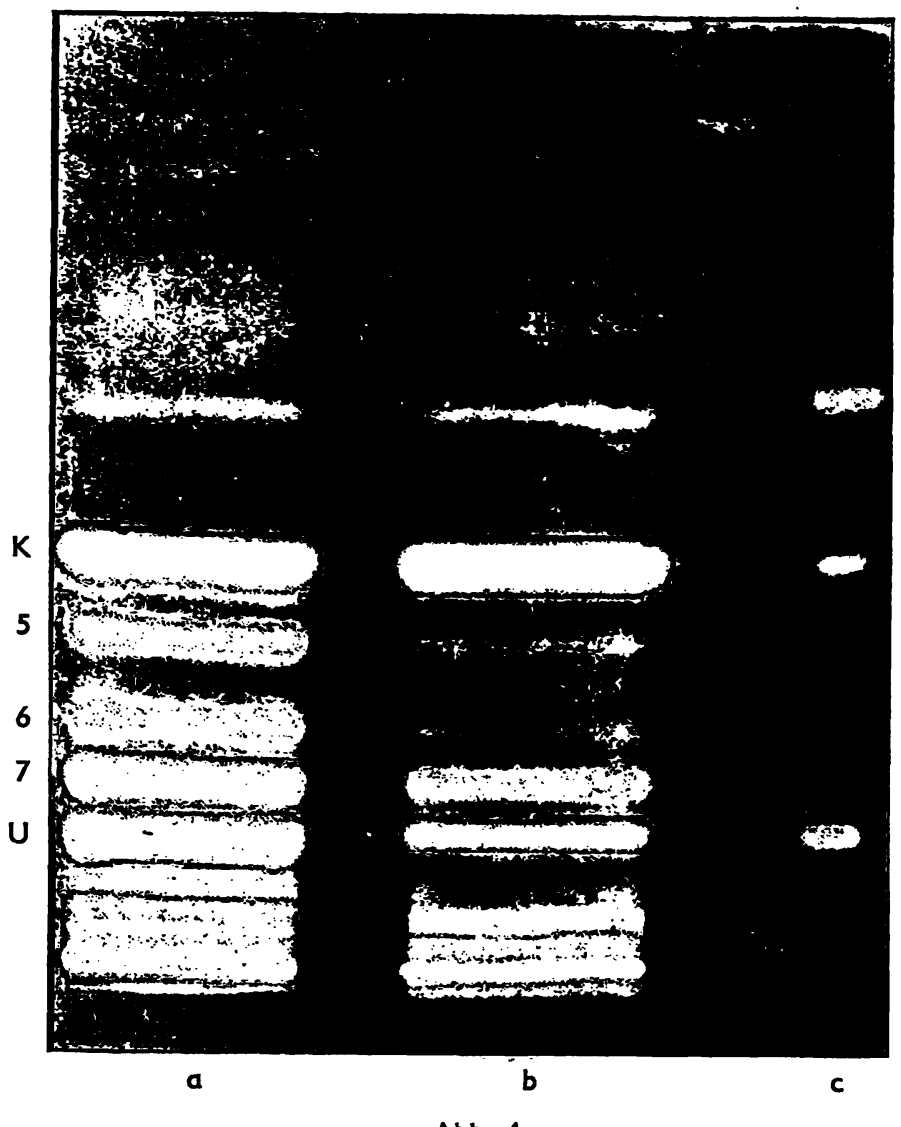

Abb. 4

Dünnschichtchromatogramm mit Urinporphyrinen des Patienten R. C. (a) mit chronischer heptatischer Porphyrie Typ C (Tab. 4, Nr. 21) experimentelle Details und Erklärung der Abkürzungen siehe gende zur Abbildung 2

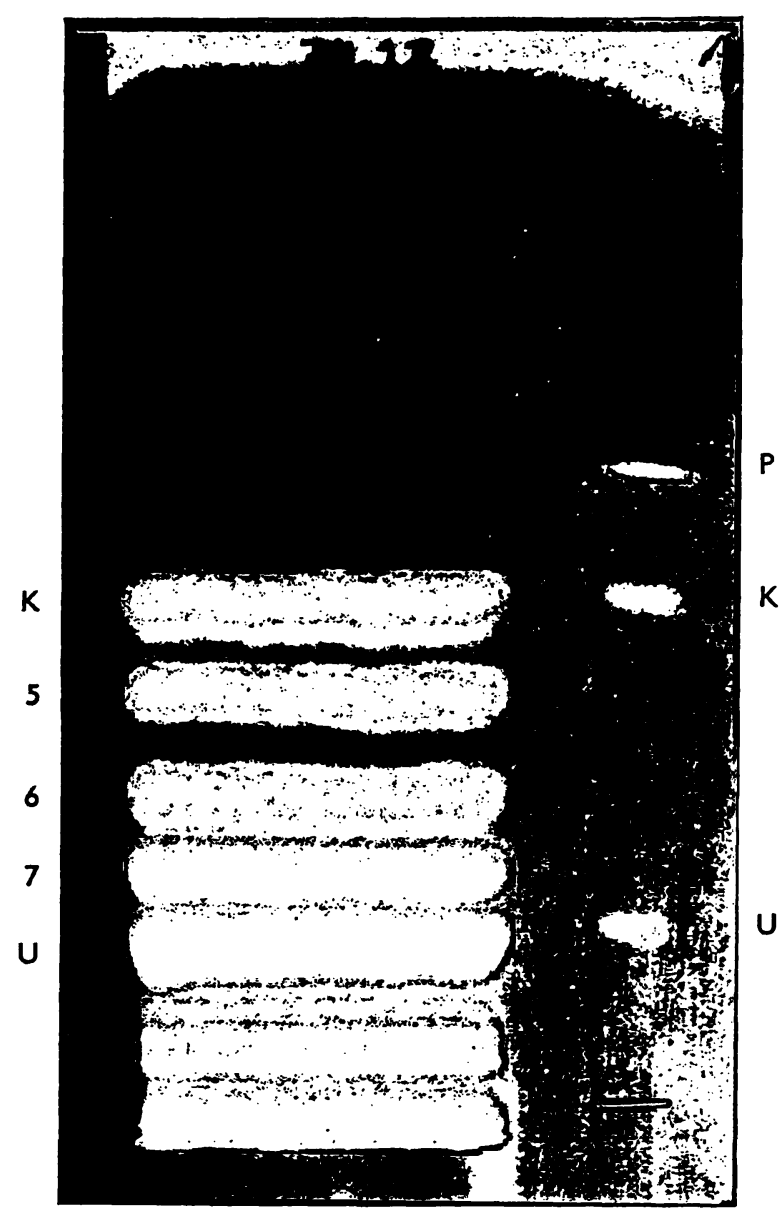

Abb. 5

Dünnschichtchromatogramm mit Urinporphyrinen des Patienten F. G. mit Porphyria cutanea tarda (Tab. 5, Nr. 27). Für experimentelle Details und Erklärung der Abkürzungen siehe Legende zur Abbildung 2

porphyrin, Heptacarboxyporphyrin und bei Konzentrationen über $30 \mu \mathrm{g} / \mathrm{g}$ auch Hexacarboxyporphyrin (Tab. 6). Der Gehalt an Proto- und Koproporphyrin war in der Leber auch dann normal, wenn ein extremer AnP stieg von Uro- und Heptacarboxyporphyrin (Patienten 26 und 32) bestand. Die Ausscheidung von $\delta$-Aminolävulinsäure und Porphobilinogen lag bei allen Patienten im Normalbereich.

$K$ Eine abnorm hohe Konzentration von Uroporphyrin mit einer gleichzeitigen Akkumulation von Heptacarboxyporphyrin in der Leber ist das erste und bleibende charakteristische Zeichen einer chronischen hepatischen Porphyrie. Ihre Einteilung in die Typen A, B und C erfolgte nach dem Ausscheidungstyp der Porphyrinurie (Abb. 2-4) und dem Porphyringehalt in der Leber. Die Konstellation der Urinporphyrine ist jedoch nicht unabhängig vom Grad der Gesamtporphyrinausscheidung. Die mittlere Gesamtporphyrinausscheidung steigt vom Typ A $(0,29 \mathrm{mg})$ über Typ B $(0,35 \mathrm{mg})$ zum Typ C $(0,62 \mathrm{mg} / 24 \mathrm{Stdn}$.) an (Tab. 2-4) und erreicht ohne weitere Änderung der Konstellation ihr Maximum bei Porphyria cutanea tarda (3,28 mg/24 Stdn.; Tab. 5). Das Verhältnis von Kopro-/Uroporphyrin ist, wie im Normalurin, $>1$ bei chronischer hepatischer Porphyrie Typ $A$, während es bei Typ $B<1$ ist (Tab. 2 und 3 ). 
Beim Vergleich der Urinporphyrine von Typ A, B und C nimmt Koproporphyrin kontinuierlich ab und Heptacarboxyporphyrin kontinuierlich zu (Abb. 2-4). So ist Koproporphyrin die zweitstärkste Komponente in Typ $\mathrm{B}$ (Abb. 3) und die drittstärkste in Typ C (Abb. 4), während umgekehrt Heptacarboxyporphyrin von der dritten Komponente in Typ B (Abb. 3) zur zweiten Komponente in Typ $\mathrm{C}$ ansteigt (Abb. 4). Damit verbunden verändert sich das Verhältnis von Kopro-/Uroporphyrin weit zugunsten von Uroporphyrin vom Typ A über Typ B zum Typ C und Typ D (Tab. 2-5, Abb. 5). Diesen Veränderungen parallel gehen der Konzentrationsanstieg der Porphyrine in der Leber (Tab. 6) und die absolute Zunahme der Porphyrinurie (Tab. 2-5). Typisch für die Leberporphyrine bei Porphyria cutanea tarda ist ein gegenüber chronischen hepatischen Porphyrien ohne klinische Symptome, insbesondere zur Abgrenzung gegenüber dem Typ $C$, höherer Anteil von Heptacarboxyporphyrin ( $33 \%, \mathrm{n}=6)$. Anhand dieses Befundes und aufgrund einer leichten Traumatisierbarkeit der Haut wurde der Patient 24 der Gruppe Typ D (Tab. 5) zugeordnet.

Der aus der charakteristischen Konstellation der Urinporphyrine bei Porphyria cutanea tarda ermittelte biochemische Index (15), dem zwei miteinander verwandte Faktoren zugrunde liegen, die sich jedoch nur in einer Richtung bedingen (Faktor $1 \rightarrow 2$ ), ist vollständig zutreffend für die chronische hepatische Porphyrie Typ C. Der erste Faktor des. Index beruht auf der relativen Verteilung der Urinporphyrine und impliziert, daß der Anteil von Uroporphyrin zwischen 45 und $80 \%$ und derjenige von Heptacarboxyporphyrin zwischen 15 und $35 \%$ der Gesamtporphyrine liegt. Sein zweiter Faktor ist dadurch gegeben, daß das Verhältnis (Uro- + Heptacarboxy-)/Koproporphyrin durch das Verhältnis Heptacarboxy-/Koproporphytin um $18-40 \%$ ansteigt. Allein dieser Faktor des biochemischen Porphyria cutanea tarda-Index ist positiv bei der chronischen hepatischen Porphyrie Typ A und Typ B.

\section{Besprechung}

Chronische hepatische Porphyrie ohne klinische Symptome ist die biochemische Manifestation einer Porphyrinsynthesestörung bei Lebererkrankung. Obgleich genetische Faktoren, die erst durch oder im Verlaufe eines Leberschadens für die Porphyrinbiosynthese relevant werden, nicht ausgeschlossen sind, betrachten wir die Stoffwechselkrankeit als „erworben" auf dem Boden einer chronischen Leberschädigung. Hinweise für einen Erbgang fanden sich bisher nicht. Nach Art und Grad der Porphyrinakkumulation in der Leber und der Porphyrinurie handelt es sich bei den chronischen hepatischen Porphyrien um intermediäre Porphyrieformen, die zwischen der sekundären hepatischen Koproporphyrinurie und der Porphyria cutanea tarda stehen (5). Die Anwendung des Porphyria cutanea tarda-Index (15) auf die vorliegenden Befunde hat die Differenzierung der chronischen hepatischen Porphyrien wesentlich geför- dert. Aufgrund der engen Verwandtschaft der chronischen hepatischen Porphyrie Typ A, B und C untereinander, die sich mit dem Porphyria cutanea tarda-Index sichern ließ und eine biochemische Progression von Typ A über Typ B und C zur Porphyria cutanea tarda (Typ D) zu erkennen gibt (16), sind Übergänge zwischen subklinisch und klinisch fließend. Das bedeutet, daß eine chronische hepatische Porphyrie in eine Porphyria cutanea tarda übergehen kann und letżtere immer von einer chronischen hepatischen Porphyrie ihren Ausgang nimmt. In einigen Fällen haben wir beobachtet, daß sich ein Typ A oder B über einen Zeitraum von einem Jahr nicht verändert. Demzufolge kann die Störung über einen längeren Zeitraum stationär und klinisch inapparent bleiben. Andererseits konnten wir eine Progredienz vom Typ $C$ in eine Porphyria cutanea tarda-Porphyrinurie von $2 \mathrm{mg} / \mathrm{l}$ unter Alkoholkonsum feststellen (Patient 22) und damit die Beobachtungen von SHANLEY und Mitarbeitern (17) bestātigen.

Aus der Sicht der Porphytiediagnostik dieses Laboratoriums $(1,4)$ ist hervorzuheben, $\mathrm{da} \beta$ die akute intermittierende Porphyrie gegenüber der Porphyria cutanea tarda selten vorkommt. Nach den vorliegenden Untersuchungen verlaufen $70 \%$ der chronischen hepatischen Porphyrinsynthesestörungen klinisch okkult (Typ A $>\mathrm{B}>\mathrm{C}$ ). Als höchstwahrscheinlich ist anzunehmen, $\mathrm{da} ß$ diese klinisch okkulten chronischen hepatischen Porphyrien die häufigste Porphyrieform nicht nur der erworbenen ("konstitutionellen", „symptomatischen") Porphyrien darstellen, sondern auch innerhalb der Gesamtgruppe der hepatischen Porphyrien (1) am häufigsten sind. Ihre milden Formen, Typ A und Typ B, die $80 \%$ der chronischen hepatischen Porphyrien ohne klinische Symptome ausmachten, können sich leicht der Diagnose entziehen, wenn der Porphyrinanstieg in Leber und Urin nicht sehr erheblich ist und bei einem chronischen Leberschaden nicht systematisch nach Quantität und Qualität einer Porphyrinurie und der hepatischen Porphyrine gefahndet wird. Im Rahmen unserer Arbeit wurde in fast allen Fällen der Verdacht auf das Vorliegen einer hepatischen Porphyrie -vom Kliniker gestellt nach subtiler Kontrolle des Leberzylinders im UV-Licht.

Bei Patient 4 und 26 korrelierte die Höhe der Porphyrinspeicherung in der Leber nicht mit dem Grad der Porphyrinurie. Wir nehmen an, daß bei den beiden Patienten besonders porphyrinreiche Leberareale getroffen wurden. Der hohe Uroporphỹringehalt in der Leber bei den Patienten mit chronischer hepatischer Porphyrie Typ C im Gegensatz sowohl zu ihrer Porphyrinauisscheidung als auch zur hepatischen Porphyrinkonzentration von vier Patienten mit Porphyria cutatanea tarda ist offenbar für jene Form charakteristisch (Tab. 4 und 6). Art und Verteilung der bei Porphyria cutanea tarda gefundenen Leberporphyrine stimmen mit der Beobachtung von DowDle und Mitarbeitern (18) überein, die neben Uround Heptacarboxyporphyrin ebenfalls einen kleinen Anteil von Hexacarboxyporphyrin nachwiesen konnten. Ein Hinweis dafür, daß ein Typ einer chronischen hepatischen Porphyrie bei einer bestimmten Lebererkrankung 
bevorzugt vorkommt, ergab sich nur für Typ B, bei dem die aggressive chronische Hepatitis im Vordergrund stand (Tab. 3).

Erstes Zeichen einer chronischen hepatischen Porphyrie ist ein geringgradiger bis mäßiger Anstieg von Uro- und Heptacarboxyporphyrin in der Leber. Dem folgt eine erhöhte Porphyrinausscheidung gemäß Typ A. Im Verlaufe einer permanent höheren Porphyrinbildung in der Leber kehrt sich im Urin das Verhältnis von Kopro-/ Uroporphyrin um: Es entwickelt sich Typ B, bei dem auch in der Leber eine im Durchschnitt höhere Porphyrinkonzentration vorliegt als bei Typ A (24 gegenüber $12 \mu \mathrm{g} / \mathrm{g}$ ). Eine weitere Erhöhung der hepatischen Porphyrinkonzentration führt zu Typ C, bei dem zwar die Urinporphyrine die charakteristische Porphyria cutanea tarda-Konstellation zeigen, der relative Anteil von Heptacarboxyporphyrin in der Leber hingegen geringer ist als bei Porphyria cutanea tarda.

Die pathobiochemische Entwicklung einer chronischen Störung der hepatischen Porphyrinsynthese ist auf dem folgenden Wege vorstellbar:

\section{Chronische Leberschädigung $\rightarrow$}

sekundäre Porphyrinurie (Koproporphyrinurie $\rightarrow$ Koprouroporphyrinurie) $\rightarrow$

chronische hepatische Porphyrie Typ A $\rightarrow$ Typ B $\rightarrow$

chronische hepatische Porphyrie Typ $C \rightleftarrows$ Porphyria cutanea tarda (Typ D).

Im Verlauf dieses Prozesses steigt die Porphyrinurie kontinuierlich an, wobei sich der Ausscheidungstẏp von $A$ bis $C$ qualitativ ändert (Abb. 2-4). Die Veränderung ist durch ein gegenläufiges Verhalten der prozentualen Anteile von Koproporphyrin- und Uro- + Heptacarboxyporphyrin von Typ A bis zu Typ D gekennzeichnet: Koproporphyrin liegt nicht unter $46 \%$ bei Typ A, nicht über $29 \%$ bei Typ B, $12 \%$ bei Typ C und 9\% bei Typ D (Tab. 2-5).
Den Befund, daß die chronische hepatische Porphyrie Typ A, B, C und die Porphyria cutanea tarda in einigen Fällen mit Lebercirrhose assoziiert waren, betrachten wir als eine Stütze der These von Levere (19), welche ihrerseits die klinischen Erfahrungen von BRUGSCH (20) bestätigt und postuliert, daß ein Leberschaden allein für die Entwicklung einer Abnormität des hepatischen Porphyrinstoffwechsels verantwortlich sein kann, die zur chronischen Porphyrie führt. Diese Abnormität imitiert insofern hereditäre hepatische Porphyrien $(21,22,23)$, als erhöhte Aktivitäten der $\delta$-Aminolävulinsäure-Synthetase, des limitierenden Enzyms der Porphyrinbiosynthese, in den Mitochondrien der cirrhotischen Leber gefunden wurden (19). Mit der Beschreibung der klinisch symptomlosen, latenten, chronischen hepatischen Porphyrien wurden die Beobachtungen BRUGSCHS und LEVEREs fortentwickelt, jedoch bleibt offen, warum in einem Teil der chronisch leberkranken Patienten die Entwicklung einer Porphyrie induziert wird, in dem anderen, größeren, hingegen nicht. Störungen im Steroidund Arzneimittelstoffwechsel bei Leberschädigungen $(24,25,26)$ können gravierende Faktoren in der biochemischen Pathogenese erworbener hepatischer Porphyrien sein.

Die klinische Bedeutung einer möglichst frühen Diagnose einer chronischen hepatischen Porphyrie ist darin zu sehen, daß ihr Verlauf durch protein- und kohlenhydratreiche Diät (27), durch Meidung von Alkohol $(17,27)$ und exogener Östrogene $(1,28)$ und vermutlich auch durch weitestgehende Meidung von Medikamenten, welche die hepatische Porphyrinsynthese zu induzieren vermögen (25), günstig beeinflußt werden kann. Außer diesen Maßnahmen steht eine Therapie, die der klinischen Manifestation infolge biochemischer Progression entgegenzuwirken vermag, noch nicht zur Verfügung.

Die Untersuchungen wurden in dankenswerter Weise durch die Deutsche Forschungsgemeinschaft (Do 134) unterstützt.

\section{Literatur}

1. Doss, M. und W. Meinhof, Dtśch. med. Wschr. 96, 1006 (1971). - 2. Doss, M., D. Look, H. Henning, P. NAwrockr, A. Schmid, W. Dölle, G. Korb und C. J. Lüders, Abstr. VIth Meeting Europ. Ass. for the Study of the Liver, London (1971). - 3. Doss, M., Hoppe-Seyler's Z. physiol. Chem. 351, 1300 (1970). - 4. Doss, M., W. Meinhof, D. Look, H. Henning, P. Nawrocki, W. Dölle, G. Strohmeyer und I. Filippini, Proc. Intern. Conf. on Porphyrin Metabolism and the Porphyrias, Kapstadt (1970), S. Afr. J. Laborat. Clin. Med. 17, 50 (1971). 5. Doss, M., D. Look und H. Henning, Klin. Wschr. 49, 52 (1971). - 6. Doss, M., Dtsch. med. Wschr. 95, 959 (1970). 7. Doss, M., diese Z. 8, 197 (1970). - 8. Doss, M., Klin. Wschr. 47, 1280 (1969). - 9. Doss, M., Z. Analyt. Chem. 252, 104 (1970). 10. Doss, M., Analytic. Biochem. 39, 7 (1971). - 11. Doss, M. und A. SChmidt, diese Z. 9, 99 (1971). - 12. ACKNER, B., J. E. Cooper, C. H. Gray, M. Kelly und D. C. Nicholson, Lancet, London 1961/I, 1256. - 13. IPPEN, H., Dtsch. med. Wschr. 88, 1788 (1963). - 14. FilippinI, L. und G. StrohmeYRR, Dtsch. med. Wschr. 94, 1577 (1969). - 15. Doss, M., W. Meinhop,
H. Malchow, K.-P. Sodomann und W. Dölle, Klin. Wschr. 48, 1132 (1970). - 16. Doss, M., Klin. Wschr. 49, 941 (1971). 17. Shanlex, B. C., S. S. ZaIl und S. M. Joubert, Brit. J. Haemat. 17, 389 (1969). - 18. Dowdle, E., P. Goldswain, N. Spong und L. Eales, Clin. Sci. 39, 147 (1970). - 19. Levere, R. D., Biochem. Med. 1, 92 (1967). - 20. Brugsch, J., Porphyrine, S. 99, Johann Ambrosius Barth, Leipzig (1959). - 21. Dowdle, E. B., P. Mustard und L. Eales, South Afr. med. J. 41, 1093 (1967). 22. Kaufman, L. und H. S. Marver, N. England J. Med. 283, 954 (1970). - 23. Tschudy, D. P., M. G. Perlroth, H. S. Marver, A. Collings, G. Hunter jr. und M. Rechcigl jr., Proc. Nat. Acad. Sci. (USA), 53, 841 (1965). - 24. Levi, A. J., SH. SHERLOCK und D. WALKER, Lancet, London 1968/I, 1275. 25. MATters DE, F., Seminars in Hematology 5, 409 (1968). 26. SiaAnley, B. C., S. S. Zail, S. M. Joubert, South Afr. med. J. 44, 293 (1970). - 27. Doss, M., P. Naivrocki, A. Schmidt, G. Strohmeyer, R. Egaring, G. Schimipf, W. Dölle und G. Kors, Dtsch. med. Wschr. 96, 1229 (1971). - 28. Thoelogrdes, A., B. J. KENNEDY und C. J. Watson, Metabolism 13, 391 (1964).

Prof. Dr. Manfred Doss Abteilung für Klinische Biochemie Hygiene-Institut der Universität D-3550 Marburg/Lahn, Pilgrimstcin 2 